\title{
The Europa Clipper Mission: Understanding Icy World Habitability and Blazing a Path for Future Exploration
}

\author{
Robert T. Pappalardo \\ Jet Propulsion Laboratory, California Institute of Technology \\ Robert.Pappalardo@jpl.nasa.gov \\ 818-354-5837
}

\section{Co-Authors:}

Tracy Becker, SwRI

Diana Blaney, JPL

Don Blankenship, UT Austin

Jim Burch, SwRI

Phil Christensen, ASU

Kate Craft, APL

Ingrid Daubar, JPL

Murthy Gudipati, JPL

Alex Hayes, Cornell U.

Sam Howell, JPL

\section{Co-Signers:}

Jacob Abrahams, UCSC

Oleg Abramov, USGS

Nicolas Altobelli, ESA

Amy Barr Mlinar, PSI

John Biersteker, MIT

Bruce Bills, JPL

Jordana Blacksberg, JPL

Mike Bland, USGS

Scott Bolton, SwRI

Christelle Briois, CNRS

Tim Brockwell, SwRI

Shawn Brooks, JPL

Lorenzo Bruzzone, U Trento

Morgan Cable, JPL

Bruce Campbell, Smithsonian

Lynn Carter, U Arizona

Tony Case, Harvard U.

Tim Cassidy, U. Colorado

Kristian Chan, UTIG

Chase Chivers, Georgia Tech

Mathieu Choukroun, JPL

Roger Clark, PSI

Corey Cochrane, JPL

Geoff Collins, Wheaton

J. Brad Dalton, JPL
Sascha Kempf, U. Colorado

Margy Kivelson, UCLA

Rachel Klima, APL

Haje Korth, APL

Erwan Mazarico, GSFC

Brian Paczkowski, JPL

Cynthia Phillips, JPL

Julie Rathbun, PSI

Trina Ray, JPL

Kurt Retherford, SwRI

Ashley Davies, JPL

Michael Davis, SwRI

Serina Diniega, JPL

Andrew Dombard, UIC

Charles Elachi, Caltech

Catherine Elder, JPL

Tucker Ely, ASU

Carolyn Ernst, APL

Leigh Fletcher, U Leicester

Antonio Genova, Sapienza U.

Yonggyu Gim, JPL

Randy Gladstone, SwRI

Chris Glein, SwRI

William Goode, U. Colorado

Thomas Greathouse, SwRI

Rob Green, JPL

Cyril Grima, UT Austin

Eberhard Gruen, U. Stuttgart

Kevin Hand, JPL

Candi Hansen, PSI

Camilla Harris, U Michigan

Hamish Hay, JPL

Paul Hayne, U. Colorado

Mark Haynes, JPL

Matt Hedman, U Idaho
Christina Richey, JPL James Roberts, APL

Abi Rymer, APL

Britney Schmidt, Georgia Tech

David Senske, JPL

Everett Shock, ASU

Elizabeth Turtle, APL

Joe Westlake, APL

Bryana Henderson, JPL

Alain Herique, IPAG

Karl Hibbitts, APL

Mihaly Horanyi, U. Colorado

Carly Howett, SwRI

Hsiang-Wen Hsu, U. Colorado

Vincent Hue, SwRI

Kynan Hughson, Georgia Tech

David Humm, APL

Terry Hurford, GSFC

Hauke Hussmann, DLR

Xianzhe Jia, U Michigan

Steven Joy, UCLA

Insoo Jun, JPL

Justin Kasper, U Michigan

Krishan Khurana, UCLA

Walter Kiefer, LPI

Randy Kirk, USGS

Wlodek Kofman, IPAG

Norbert Krupp, Max Planck

Bill Kurth, U Iowa

Nic Kutsop, Cornell U.

Yves Langevin, U Paris-Sud

Justin Lawrence, Georgia Tech

Erin Leonard, JPL 
Jiang Liu, UCLA

Jonathan Lunine, Cornell U.

Adrienn Luspay-Kuti, APL

Kathleen Mandt, APL

Jean-Luc Margot, UCLA

Marco Mastrogiuseppe, DII

Alexandra Matiella Novak, APL

Tom McCord, Bear Fight Inst. Alfred McEwen, U Arizona

Melissa McGrath, SETI

Bill McKinnon, Washington U.

Ralph McNutt, APL

Mike Mellon, Cornell U.

Mohit Melwani Daswani, JPL

Heather Meyer, APL

Kelly Miller, SwRI

Philippa Molyneux, SwRI

Jeff Moore, Ames

Olivier Mousis, CNRS

Alina Moussessian, JPL

Scott Murchie, APL

Neil Murphy, JPL

Edward Nerney, U. Colorado

Francis Nimmo, UCSC

Tom Nordheim, JPL

Chris Paranicas, APL

Ryan Park, JPL

G. Wes Patterson, APL

Carol Paty, U. Oregon
Sylvain Piqueux, JPL

Jeff Plaut, JPL

Dirk Plettemeier, TU Dresden

Antoine Pommerol, U. Bern

Frank Postberg, FU Berlin

Louise Prockter, LPI

Lynnae Quick, GSFC

Ujjwal Raut, SwRI

Christine Ray, SwRI

Carol Raymond, JPL

Thomas Richter, UTIG

Kirtland Robinson, WHOI

Lorenz Roth, KTH

Elias Roussos, Max Planck

Chris Russell, UCLA

Tara Salter, Imperial College

Joachim Saur, U Cologne

Kirk Scanlan, UTIG

Juergen Schmidt, U Oulu

Dustin Schroeder, Stanford

Jennifer Scully, JPL

Melissa Sedler, ASU

Frank Seelos, APL

Mark Sephton, Imperial

Jonas Simolka, U. Stuttgart

Vishaal Singh, ASU

Jim Slavin, U Michigan

H. Todd Smith, APL

Jason Soderblom, MIT

Krista Soderlund, UT Austin
John Spencer, SwRI

Elizabeth Spiers, Georgia Tech

Ralf Srama, U. Stuttgart

Andrew Steffl, SwRI

Gregor Steinbrugge, Stanford

Alan Stern, SwRI

Zoltan Sternovsky, U Colorado

Michael Stevens, Harvard U.

Robert Strangeway, UCLA

Marshall Styczinski,

$\mathrm{U}$. Washington

Sarah Sutton, U. Arizona

Ben Teolis, SwRI

Nicolas Thomas, U Bern

David Thompson, JPL

Jay Thompson, JPL

Gabriel Tobie, LPG Nantes

Paolo Tortora, U. Bologna

Zachary Ulibarri, U. Colorado

Steve Vance, JPL

Janet Vertesi, Princeton

Michaela Villarreal, JPL

J. Hunter Waite, SwRI

Ben Weiss, MIT

Paul Withers, Boston U.

Natalie Wolfenbarger, UTIG

Danielle Wyrick, SwRI

Duncan Young, UT Austin

Mikhail Zolotov, ASU 
This paper discusses the science expected to be achieved by the Europa Clipper mission,

in order to provide context for the future exploration of Europa and other icy worlds. In addition, we discuss how the science will be achieved as we strive to operate as "One Team."

\section{Introduction: Science Goal and Objectives of the Europa Clipper Mission}

Europa is a complex geophysical and geochemical system with great astrobiological potential, and host to a wide range of processes relevant to understanding the Solar System's ocean worlds. These processes include: ice tectonics, cryomagmatism, impact cratering, mass wasting, ice convection, tidal deformation and heating, surface-plasma chemical and physical interactions, exospheric and ionospheric processes, magnetospheric interactions, and possibly plume dynamics. It is also a key target for astrobiological exploration because it may contain the “ingredients" for life: liquid water, bioessential elements, and the chemical energy to power metabolism; moreover, these ingredients may have persisted since early Solar System history, providing opportunity for life to emerge. This astrobiological potential has made Europa exploration a top priority of the first two planetary science decadal surveys, ultimately giving rise to the Europa Clipper mission in 2015. Expected to arrive at Jupiter near the end of the coming decade, this mission will result in discoveries and knowledge that will feed forward and inform future ocean world missions, so its potential should factor into the present planetary decadal planning.

As shaped by prior decadal surveys, the science goal of the Europa Clipper mission is to explore Europa to investigate its habitability. Addressing this goal are three science objectives:

(1) Characterize the ice shell and any subsurface water, including their heterogeneity, ocean properties, and the nature of surface-ice-ocean exchange;

(2) Understand the habitability of Europa's ocean through composition and chemistry;

(3) Understand the formation of surface features, including sites of recent or current activity, and characterize high science interest localities.

The search for and characterization of recent activity is cross-cutting and may manifest as plumes, thermal anomalies, or surface geological and/or compositional changes.

To address the science of the Europa Clipper mission, a highly capable suite of instruments that comprise the mission's scientific payload is now under development. This payload includes five remote sensing instruments ${ }^{1}$ that will observe the wavelength range from the ultraviolet through radio (radar) and four in situ instruments that will measure neutral gases, magnetic fields, dust particles, and plasma. In addition, gravity and radio science will be conducted through tracking of the spacecraft's telecommunication signal during Europa flybys, and valuable scientific data can come from the spacecraft's radiation monitor engineering subsystem. To achieve the goal and objectives of the mission, combining multiple data sets will provide insights into the geophysical processes that affect this icy world. Toward this end, Europa Clipper adopts a "OneTeam” philosophy, which may serve as a model for future flagship missions.

\footnotetext{
${ }^{1}$ In this paper, "instrument” refers to the hardware, while "investigation” refers to the collection of science that an instrument is intended to achieve. The Europa Clipper has 9 science instruments (if the EIS camera suite is counted as one), while science investigations can also be achieved via the telecommunications and radiation monitor engineering subsystems.
} 


\section{Science Investigations of the Europa Clipper Mission}

\subsection{Remote Sensing Investigations of Europa}

Europa Clipper's remote sensing instruments provide the capability for in-depth study of Europa's interrelated processes. They will be employed to understand the detailed processes associated with ocean-ice shell interactions, transport within the ice shell, tectonism, cryomagmatism, cratering, and recent activity. Below we summarize the key characteristics of each remote sensing instrument and associated investigations, in wavelength sequence.

Europa Ultraviolet Spectrograph (Europa-UVS). Europa-UVS is the mission's primary plume finder. The instrument will search for plumes by spatial and spectral imaging of UV emissions (airglow) and transmission (stellar occultations) in the wavelength range of 55-210 $\mathrm{nm}$ and a spectral resolution of $0.6 \mathrm{~nm}$. Data to be collected by Europa-UVS will be used to interrogate the composition and chemistry, sources and sinks, and structure and variability of the tenuous Europa atmosphere. In addition, it will be possible to constrain surface composition and microphysics, and relationships to endogenic and exogenic processes. Europa-UVS data will also permit a better understanding of Europa's plasma environment, notably the energy and mass flow into Europa's atmosphere, neutral cloud, and plasma torus.

Europa Imaging System (EIS). EIS is composed of a narrow-angle camera (NAC) and a wideangle camera (WAC), each with both framing and pushbroom capability, along with stereo and color imaging capability. Together, the EIS camera suite will be used to map Europa nearglobally at better than $100 \mathrm{~m}$ resolution and capable of imaging almost any point on the surface at better than $20 \mathrm{~m}$ resolution. EIS images and topography data will build a foundation for geologic mapping and for testing geophysical models to understand the formation of surface features and their implications for subsurface water. The WAC provides topographic data to characterize surface clutter in REASON shallow subsurface profiles. NAC very high-resolution imaging ( $\leq 1$-m pixel scale) will characterize the surface at the meter scale, addressing local-scale regolith processes. Distant imaging by EIS will search for active plumes, and color imaging will search for surface changes from previous Voyager and Galileo imaging and during Europa operations. NAC science measurements are prioritized in the mission's formal requirements over those from the WAC. Also, while not part of Europa Clipper's formal objectives, EIS and other Europa Clipper data can be used to characterize the science value and engineering safety of sites for a possible future landed mission.

Mapping Imaging Spectrometer for Europa (MISE). Operating in the 0.8-5.0 $\mu \mathrm{m}$ wavelength range, MISE data will be used to assess the habitability of Europa's ocean through the examination of the inventory and distribution of surface compounds, including materials relevant to astrobiology. MISE data will be used to identify and map the distributions of organics, salts, acid hydrates, water ice phases, altered silicates, and radiolytic compounds. It also has the potential to detect sufficiently warm thermal anomalies.

Europa Thermal Imaging System (E-THEMIS). The E-THEMIS instrument data can be used to detect and characterize thermal anomalies that may indicate recent or current activity. Thermal inertia information derived from E-THEMIS temperature measurements can be used to characterize regolith particle size, block abundance, and subsurface layering.

Radar for Europa Assessment and Sounding: Ocean to Near-surface (REASON). Data from the REASON instrument will permit mapping of the vertical structure of Europa's icy shell and the 
associated search for subsurface water and brine. REASON will also be used to search for the deeper ice-ocean interface, while characterizing the ice shell's global thermophysical structure. This will provide insights into material exchange among the ocean, ice shell, surface, and atmosphere. Derived physical properties of surface permittivity and roughness will support characterization of sites at local scales, including for a potential future lander.

\subsection{In situ Investigations of Europa}

The influence of the Jovian space environment on Europa is multifaceted, and observations of moon-magnetosphere interaction by Europa Clipper's instruments will provide an understanding of the satellite's interior structure and compositional makeup, among the many advances. The variability of Jupiter's magnetic field at Europa induces electric currents within the moon's conducting ocean layer, the magnitude of which depends on the ocean's location, extent, and conductivity. Europa is also embedded in a flow of co-rotating plasma, which continuously impacts and sputters the surface to produce the moon's tenuous atmosphere. In addition, micrometeorite impacts eject particles off the surface to wrap Europa in a cloud of dust. The neutral atmosphere is readily ionized by energetic particles to produce an ionosphere, which gives rise to current systems electromagnetically connecting Europa to Jupiter; neutral and ionized volatiles and particles define Europa's exosphere. Summarized below are some highlights of the moon-magnetosphere interaction science that we seek to unravel with the Europa Clipper mission and the primary instruments involved in these investigations. We also briefly summarize gravity / radio science and radiation science.

Europa Clipper Magnetometer (ECM). The facility-managed Europa Clipper Magnetometer (ECM) will measure magnetic fields generated by currents induced in Europa's subsurface ocean and the electromagnetic coupling of the moon to Jupiter. Jupiter's tilted dipole magnetic field and Europa's eccentric orbit expose the moon to time-varying magnetic fields inducing eddy currents in the ocean. By inferring the induced field response at multiple frequencies, the ice shell thickness and the ocean layer thickness and conductivity can be uniquely determined for a range of likely parameters. The ECM consists of three fluxgate sensors mounted on an 8.5-mlong boom, with its control electronics hosted in a vault shielding it from radiation damage. The use of three sensors allows for dynamic removal of higher-order spacecraft-generated magnetic fields on a boom that is short compared with the spacecraft dimensions.

Plasma Instrument for Magnetic Sounding (PIMS). The PIMS instrument will measure ions and electrons in Europa's ionosphere and Jupiter's magnetosphere, to quantify magnetic fields associated with electric currents driven by dynamic plasma pressure gradients, which are produced by the interaction of corotating Jovian plasma with Europa and its atmosphere. The PIMS instrument consists of two sensors each hosting two Faraday cups, each with a $90^{\circ}$ field of view. The instrument measures electrons and ions with energies of up to $2 \mathrm{keV}$ and $6 \mathrm{keV} / \mathrm{q}$, respectively, with an energy resolution of $<15 \%$ and a temporal resolution of $1-4 \mathrm{~s}$. From the energy distribution functions, the ion density, temperature, and velocity as well as the electron energy and temperature can be inferred.

Mass Spectrometer for Planetary Exploration (MASPEX). The MASPEX instrument will measure trace neutral species, to determine the composition of Europa's atmosphere. The atmosphere is produced by particles sputtering off the surface and may be augmented by possible contributions from plumes. MASPEX data will be used to determine the distribution of major volatiles (such as $\mathrm{H}_{2} \mathrm{O}, \mathrm{H} 2, \mathrm{O} 2$, and $\left.\mathrm{CO}_{2}\right)$ and key organic compounds $\left(\mathrm{CH}_{4}, \mathrm{C}_{2} \mathrm{H}_{2}, \mathrm{C}_{2} \mathrm{H} 4\right.$, 
C2H6, $\mathrm{HCN}$, and $\mathrm{CH} 3 \mathrm{OH}$ ) with respect to latitude and longitude, altitude, and local solar time. The data will permit us to distinguish endogenic and exogenic sources. MASPEX is a multibounce time-of-flight mass spectrometer for species with atomic mass ranging from 2 to $500 \mathrm{u}$. The instrument's high mass resolution $\mathrm{m} / \Delta \mathrm{m}$ of greater than 17,000 enables measurement of individual fragmented ions generated by electron impact ionization of parent molecules. It is planned that MASPEX will be equipped with a cryotrap for sample storage of trace species, for subsequent analysis near apoapsis, an operational environment that has a significantly lower background radiation-noise environment than that encountered during the Europa flybys. NASA has deemed MASPEX to have a higher risk classification than the rest of the science payload.

Surface Dust Analyzer (SUDA). The SUDA instrument will permit analysis and mapping of the chemical composition of particles ejected from Europa's surface by hypervelocity impacts of micrometeoroids. The dust particles have a typical size of $0.5-1 \mu \mathrm{m}$ and exhibit a near-isotropic distribution and only weak temporal variations. SUDA will also measure particles from exogenous sources, such the other Galilean satellites, notably Io. SUDA is a time-of-flight mass spectrometer for atomic and molecular species with mass ranging of up to $500 \mathrm{u}$ ionized during hypervelocity impact of ice/dust particles on SUDA's target, and is capable of detecting up to 100 ejecta particles per second.. Owing to the deterministic nature of the ballistic trajectories of the ejected particles, the composition measurements at spacecraft altitude can be correlated with geologic features on the surface. It has been experimentally demonstrated that trace amounts of complex organic species, such as amino acids, embedded in ice grains with abundances $<0.1$ ppm can be detected. In addition to micrometeorite ejecta, SUDA could measure the makeup of potential plumes by directly sampling microscopic particles.

Gravity and Radio Science (G/RS). NASA has recently selected a science team to plan, process, and analyze gravity and radio science (G/RS) data through tracking of the spacecraft's telecommunication signal during Europa flybys. The selected geodetic investigations include topics of Europa's gravity anomalies in the ice shell and silicate interior, time-varying gravity field and tides, planetary orientation, and ephemeris and orbital evolution. Moreover, radio occultations will provide information about Europa's ionosphere.

Radiation Monitoring System (RADMON). RADMON is an engineering subsystem system that consists of a charge monitor stack and eight dosimeters distributed around the spacecraft. It will permit monitoring of the radiation environment at Jupiter beneath different thicknesses of shielding, to understand the instantaneous dose rate and accumulated ionizing dose over time. RADMON will permit measurements of energetic particles up to $40 \mathrm{MeV}$, complementing PIMS measurements.

\section{Mission Concept}

The current Europa Clipper mission design consists of $~ 40-50$ low-altitude flybys of Europa while the spacecraft is in orbit about Jupiter. The flybys extend over a $~ 3.8$-year period. The altitudes of closest approach typically range from $25 \mathrm{~km}$ to $100 \mathrm{~km}$, and up to $2400 \mathrm{~km}$. The tour is divided into two principal campaigns, first making flybys that concentrate on the sunlit antiJovian hemisphere, followed by observations primarily of the sunlit sub-Jovian hemisphere. There are also several night-time flybys included in the tour design. The flybys occur over a wide range of latitudes and longitudes, and they are also well-distributed in true anomaly of Europa's orbit, local solar time, and System III Jovian longitude. The tour is thus well-suited for 
characterization of Europa's surface, ocean, ice shell, atmosphere, and ionosphere, and interactions with Jupiter's magnetosphere.

\section{Europa Clipper One-Team Structure}

To understand Europa and its potential habitability, the science must be synergistic, with the suite of instruments and investigations working together, each making contributions toward addressing the science objectives and goal. In this section we discuss the structure of the science team and how it works as a single entity to advance our knowledge of this icy world.

\subsection{Flight System Operability}

Fundamental to the science team working together as one is the premise that the flight systemwhich comprises the spacecraft and the instrument payload-enables the instruments to operate simultaneously. Deliberate care was taken to design the Europa Clipper flight system such that all of the Europa Clipper science instruments can be operated simultaneously, for each and every Europa flyby. This approach presents challenges in the areas of energy usage and power storage, data rate into the spacecraft's storage system, and in the mutual placement of the instruments. This commitment is fundamental to the Europa Clipper team operating as one by diminishing the conflict over the mission's most precious resource: time.

The mission lifetime is limited by radiation, so use of only some instruments on a given flyby would be inefficient. Moreover, an operational plan that is relatively simple and repeatable reduces costs by simplifying planning and execution. But just as important, limiting competition for the key resource of close-approach observation time allows the science team to work cooperatively toward common scientific ends, rather than debating the merits of limited instrument usage. One challenge has been achieving gravity and radio science during flybys, successfully addressed by situating 2 low-gain antennas and 3 fan-beam antennas around the spacecraft, in configurations such that at least one has a line-of-sight to Earth during about 2/3 of the Europa flybys. Electromagnetic compatibility of the flight system, including instruments, is a significant challenge ahead.

\subsection{Science Team Structure and Composition}

The Europa Clipper Science Team is composed of members of its 10 Investigation Teams (consisting of a Principal Investigator (PI) or Team Leader (TL), Co-Investigators [Co-Is], and Affiliate members (including Professional, Postdoctoral, and Graduate Student Affiliates) and the Project Science Team (Project Scientist and Deputies, Project Staff Scientists, and Investigation Scientists). In the future, it is planned that the mission will include Participating Scientists from the scientific community, solicited and selected by NASA. All science team members have the opportunity to participate in Project Science Group (PSG) meetings (i.e., science team meetings) and occasional cross-investigation science meetings (such as plume workshops).

The Europa Clipper mission PIs and Co-Is were competitively selected by NASA in 2015. PIs each proposed a science investigation implemented through a specific instrument, along with a team of Co-Is to complete the science investigation. At the time of selection, the 9 PI-led instrument/investigation teams included 96 unique individuals, with 16 women (17\%). Of the 78 scientists from US institutions, 15 are women (19\%). Very few members of the competitively selected science team are people of color. The addition of Affiliate and Project Science Team 
members on this long-duration mission has increased the ratio of women science team members to approximately $25 \%$. Team members proactively seek out social-science-backed best practices and engage in regular discussions so as to continue to improve inclusivity and diversity within the team. Our One-Team philosophy strives to provide team members with an inclusive environment, empowering them to be an integral part of the Europa Clipper Mission.

\subsection{Thematic Working Groups and Focus Groups}

Science implementation of the Europa Clipper mission is supported by a set of four Thematic Working Groups (TWGs) that represent the mission's science goal and objectives: Habitability, Interior, Geology, and Composition. The TWGs are designed to provide a high-level, crossinstrument and cross-discipline, objective-driven science perspective. All Europa Clipper Science Team members have the opportunity to participate in the strategic and tactical science planning efforts through the TWGs. This aids in ensuring that the goal and objectives of the mission are met through achieving high-quality integrated science, maximizing scientific impact.

Focus Groups address key topical areas that are especially important and relevant to the science team. The Plume Focus Group is charged with studying and discussing science requirements, policies, and trades as they affect the mission's science with regard to potential plumes and related phenomena at Europa. The Radiation Focus Group promotes discussion among the science team of issues related to radiation ( $>100 \mathrm{keV}$ electrons and ions) and addresses and communicates radiation-related issues that arise from the science team. The Reconnaissance Focus Group considers and advocates for reconnaissance data sets that best enable a potential future landing on the surface of Europa.

Rotation of one of the two co-chairs of the science team's TWGs and Focus Groups every few years aims to broaden participation, foster diversity at the project leadership level, and promote a range of ideas. This system can promote junior team members and members of underrepresented groups to positions that provide valuable leadership experience. These scientists return to the greater science team as demonstrated leaders, bolstering the knowledge and experience base of the whole science team.

\subsection{One Team Philosophy}

Our “One Team” philosophy prioritizes synergistic science by bridging across the individual instrument-based investigations, while promoting collaborations among members of the Europa Clipper science team. Each of the Europa Clipper individual instruments will be used to investigate Europa and its environs, finding critical clues about how Europa works as a planetary body. In combining and assessing the datasets from each instrument's experiments, we can collectively gain clarity into Europa's mysteries. As is commonly true in science, it is at the overlapping boundaries of our subfields that the greatest insights and discoveries will be made. Integrated science celebrates our individual expertise, challenges our assumptions, breaks through our limitations, and expands our intellectual boundaries. Associated visibility brings trust, promotes partnerships, and enhances personal relationships. These aspirations are the inherent basis for functioning as one science team.

Rules of the Road. The Europa Clipper Science Team "Rules of the Road" document provides team policies on data sharing, publications, professional code of conduct, and science team responsibilities for the team. This document is intended to provide open, transparent, and equitable operating rules for the Europa Clipper Science Team for the duration of the Europa 
Clipper project, to enable strong working relationships and to ensure that the highest quality science is delivered from the project. All Europa Clipper Science Team members are required to abide by and uphold the policies and practices described in that document. The Rules of the Road document includes the Europa Clipper Science Team's Code of Conduct, which also describes enforcement and consequences of non-compliance.

Data Sharing. Uninhibited sharing of data among members of the Europa Clipper Science Team is essential if the highest quality interdisciplinary science is to be accomplished. Data sharing enables multiple datasets and multiple perspectives to be considered in concert and can lead to unexpected insights and better understanding of the processes operating at Europa. Therefore, the Europa Clipper Science Team's data sharing policies aim to encourage cross-instrument, crossinvestigation, and multi-expert interactions at all levels, to support development of working relationships and partnerships, and engender the trust necessary to enable emerging collaborations across the project. The Europa Clipper project is also committed to ensuring usability and timely sharing and distribution of data to the science community and the public.

Publication Policies. As the end products of our scientific investigations, it is important that science results be shared with the science community, NASA, and the public as soon as feasible. The full Europa Clipper Science Team is presented with the opportunity to contribute to any multiple-investigation scientific publications in ways appropriate and practical, notably during TWG interactions. Outlines or rough-drafts of all manuscripts are posted on a team website when they are sufficiently mature to be written up as a journal paper, to ensure communication and visibility for ongoing projects, and mature drafts of all manuscripts are posted and announced before they are to be submitted to ensure visibility among team members.

\section{Conclusion}

Taken together, the Europa Clipper mission's robust investigation suite can be used to test hypotheses and enable discoveries relevant to the interior, composition, and geology of Europa, thereby addressing the potential habitability of this intriguing ocean world, in line with recommendations from the previous planetary science decadal surveys.

The one-team philosophy of the mission and associated visibility brings trust, promotes partnerships, and enhances personal relationships. Such an approach should be considered for future strategic missions. For a long-duration mission such as Europa Clipper, one could say that it's all about the journey; therefore, we strive to make this journey a great one, for the good of the science, our mission, and each other.

\section{Acknowledgements}

This work was supported by NASA through the Europa Clipper Project. A portion of this research was carried out at the Jet Propulsion Laboratory, California Institute of Technology, under a contract with the National Aeronautics and Space Administration. 